\title{
Emotions processing in amyotrophic lateral sclerosis and behavioral variant of frontotemporal dementia-correlation with executive functions
}

\author{
Paulina Pasinska', Lukasz Krzywoszanski ${ }^{2}$, Monika Ostrowska ${ }^{1,3}$, Aleksandra Szyper-Maciejowska', Katarzyna \\ Kowalska', Tomasz Dziedzic ${ }^{4}$, Aleksandra Klimkowicz-Mrowiec ${ }^{4, t}$
}

\begin{abstract}
The ability to decode emotions in amyotrophic lateral sclerosis (ALS) and fronto-temporal dementia (FTD) still requires research. The evidence demonstrates impairment of emotion recognition in behavioral variant FTD (bvFTD); data regarding emotions decoding in ALS are discrepant. Ability to decode emotions may depend on different variables, among them executive functions. The aim of this study is to compare patients with bvFTD, ALS and controls on emotions decoding ability. The second aim was to explore correlation between executive impairment and emotional decoding abilities. This study contained 26 patients with bvFTD, 42 with ALS and 31 control subjects with the same age, education and disease severity. Patients completed neuropsychological tests, and emotional recognition tasks. Patients with bvFTD decoded emotions worse than ALS and controls. More significant associations between the ability to decode emotions and executive dysfunction were found in ALS than in bvFTD patients and controls.
\end{abstract}

\section{Keywords}

Amyotrophic lateral sclerosis, Frontotemporal dementia, Emotions decoding abilities, Executive functions

\begin{abstract}
Introduction
Amyotrophic lateral sclerosis (ALS) is a neurodegenerative disease, characterized by a progressive degeneration of the lower motor neurons in the anterior horn of the spinal cord, brainstem motor nuclei and loss of upper motor neurons in the motor cortex. Clinically, ALS produces physical impairment: progressive weakness, muscle wasting, fasciculation, spasticity, difficulty with breathing and swallowing [1]. For a very long time the cognitive abnormalities in ALS were unrecognized, but
\end{abstract}

the concept that cognitive and behavioral dysfunctions in ALS are rare, can no longer be supported. According to previously published studies about $50 \%$ of ALS patients have some cognitive impairment, and $10-15 \%$ can be diagnosed with frontotemporal dementia (FTD) [2-5].

Frontotemporal dementia is a neurodegenerative disease, associated with atrophy of the frontal and/or anterior temporal lobes. It is a clinically and pathologically heterogeneous syndrome. The most common presentation is a change

'Department of Neurology, University Hospital, Krakow, Poland

${ }^{2}$ Chair of Psychology, Pedagogical University, Krakow, Poland

3John Paul II Hospital, Neurology Department, Krakow, Poland

${ }^{4}$ Chair of Neurology, Faculty of Medicine, Jagiellonian University Medical College, Krakow, Poland

${ }^{\dagger}$ Author for correspondence: Aleksandra Klimkowicz-Mrowiec, Chair of Neurology, Faculty of Medicine, Jagiellonian University Medical College, Krakow, Poland. Tel: +48 1242486 00; Fax: +48 1242486 26, email: Aleksandra.Klimkowicz@mp.pl 
in personality and impaired social conduct (the behavioral variant FTD (bvFTD)). Less frequently patients present with language problems (primary progressive aphasia) [5]. Behavioral variant is the most common fenotype of the FTDs. It is characterized by gradual deterioration of personality presenting as apathy or disinhibition, reduced empathy and insight, poor self-care, mood changes and executive dysfunction [6].

Although ALS and FTD most often occur separately, genetic [7], pathological [8] and clinical [9] research has hypothesized that changes observed in ALS and FTD lie on a common line where solely motor system involvement is observed on one end, and behavioral and cognitive changes characteristic for FTD is on the other end, yet others have not supported this notion [10].

The pathological changes characteristic for bvFTD and ALS involve similar areas of the brain with the extent of the atrophy being different; the most advanced in FTD, followed by ALSFTD, ALS with cognitive deficit and lastly ALS without cognitive deficit the least [11].

A continuum between ALS and bvFTD in cognition and behavior was postulated based on observations of prefrontal dysfunction in a significant proportion of patients with ALS.

Behavioral changes can be a consequence of deficit recognition of basic emotions. A network of limbic structures that include the amygdala, insula and orbitofrontal cortex, which are activated in response to emotional stimuli, is affected in bvFTD [12,13].

Previous studies on emotional perception have showed inconsistent data, although most studies have found deficits in non-demented or FTDcombined ALS patients [14-16]. However the reason why ALS patients have problems to recognize emotions is poorly understood.

The ability to recognize emotions in others is a complex process and involves many different brain circuits. This deficit may be a consequence of a dysfunction of the orbitofrontal cortex or disruption in the prefrontal-limbic structure [16].

Recognizing emotions is related to the ecological value of the stimuli and the channel through which the emotion is primarily communicated (face, hand gestures, body pose), [17]. Carrying emotional stimuli through different channels at the same time, may simplify decoding ability.

Evidence, from studies assessing emotional decoding ability in patients with different psychiatric populations (schizophrenia, affective diseases, pathological behavior) suggests, that executive cognitive capacities and emotional processing may be related [18-21]. Decoding emotions may be dependent on the efficiency of executive system especially when there is a demand to control and process complex information.

Direct comparison between FTD and ALS and healthy subjects regarding emotional decoding abilities are scarce and obtained results have also been inconsistent [14,22].

Therefore, our first aim was to compare the performance of healthy subjects with ALS and by FTD patients, on emotional decoding abilities using simple or complex stimuli.

We hypothesized that emotional decoding ability of ALS patients would decline compared to controls while their performance in emotional decoding would be better than in patients with by FTD. We also expected the same pattern of differences between groups in executive functioning.

The second aim was to identify relationship between executivemeasures of neuropsychological evaluation, and ability to distinguish emotions within each group.

We hypothesized that executive impairment may negatively affect recognition of emotional stimuli.

\section{Material and Methods}

\section{- Participants}

We studied a sample of 42 (63.39 years old (SD 6.57, range 50-77)) patients (27 females) with ALS, 26 (61.38 years old (SD 7.84, range 48-78)) (17 females) with bvFTD and 31 (61.23 years old (SD 8.01, range 49-74)) (22 females) controls. The average number of years of education was 12.50 (SD 3.72, range 6-18) for ALS patients, 14.08 (SD 3.50, range 7-22) for bvFTD patients and 13.52 (SD 2.58, range 10-18) for control subjects.

Patients with ALS and bvFTD were recruited from the Outpatient Clinic in Neurology Department at the Jagiellonian University Hospital. Each patient followed a medical, neurological, and neuropsychological examination; was 

correlation with executive functions

interviewed with a close informant; underwent laboratory testing; and computed tomography or magnetic resonance imaging.

On neuropsychological examination the following methods were used: executive functions measures (verbal fluency (subtests from Mattis Dementia Rating Scale (MDRS) [23]), non-verbal fluency [24], Trail Making Tests part B [25], Tower of London [26], Stroop Test [27]), orientation (orientation items of MoCA [28]), attention and concentration (digit span, subtest of MoCA, Trail Making Test part A [25]), reasoning (calculation, arithmetic and verbal problem solving) [24], constructional and visuospatial functions (subtests of MDRS, construction of MoCA, Rey-Osterrieth Complex Figure Test (ROCFT) ) [24], verbal memory (California Verbal Learning Test Polish version (CVLT) [29]), nonverbal memory (Rey-Osterrieth Complex Figure Test), language (repetition, naming), gestural praxis (subtests of MDRS, pantomiming of object use without objects), gnosis (identification of objects and naming of objects' pictures) [24], writing (subtests of MDRS).

Diagnosis of bvFTD was made according to the recent consensus diagnostic criteria [30]. Staging of the disease was performed using Frontotemporal Dementia Rating Scale (FRS) [31]; only patients with mild and moderate stage of the disease were included. All included patients had at least a 2 years observational period to exclude patients with different variants of FTD, frontal variant of Alzheimer's disease or patients with "phenocopy" FTD (syndrome with behavioral changes characteristic of bvFTD, but without abnormalities on structural or functional neuroimaging and without decline in activities of daily leaving over time) [32].

The clinical diagnosis of ALS was established according to the revised El Escorial criteria [1]. Staging of the disease was performed using the ALS Functional Rating Scale-Revised [33]. Only patients who could make either a verbal or written response were included into the study. Patients who were classified as ALS-FTD were excluded from the study.

The control group included unrelated caregivers or volunteers that were consecutively recruited from the general population. They had no apparent neurological disease or psychiatric syndrome, cerebrovascular diseases or severe functional limitations.
All participants were interviewed to obtain scores on the 15-item Geriatric Depression Scale [34], those who scored more than 5 points were excluded from the study.

All participants provided informed consent to participate in the study, which the local ethical committee approved.

\section{- Experimental procedure}

Emotional recognition task is a paradigm for measuring the recognition of six basic facial emotional expressions: anger, disgust, fear, happiness, sadness, and surprise.

In our study each participant completed a session which consisted of 6 different sets of cartoonlike drawings presenting 7 different stimuli (6 basic emotions: happiness, sadness, fear, disgust, anger, surprise and one neutral stimuli). The following sets progressively increased in their level of complexity. Examples of stimuli used for emotional decoding are presented elsewhere [35]. The first two sets presented pictures of facial expressions only: (1) icon - simple emotion icon, (2) boy - detailed drawings of human facial expression. The following 3 sets presented humans with various facial expressions and gestural poses (3) girl, (4) woman and (5) boy drawings of people in various casual situations. In the last set, instead of using humans emotions were presented by personified birds. The first and the second sets investigated the facial channels of emotional communication only, while the third to sixth sets illustrated both facial and gestural channels of emotional expression. In all six sets, stimuli were presented in random order and pictures were presented one at a time. Each patient was provided with a cue sheet with labels of all stimuli and asked to pick the one that they thought best matched the drawing. The drawings within sets two to five portrayed scenarios that were the most similar to scenarios present in the individual's natural environment. The numbers of correct recognition in each set were collected.

The basic assumptions underlying construction of emotional decoding task were confirmed by Klimkowicz-Mrowiec, et al. [36]. The scores obtained in consecutive sets changed depending on the level of complexity and ecological value of the emotional stimuli. In patients with amnestic type of mild cognitive impairment, patients with mild Alzheimer dementia and control subjects scores in sets 3-5, containing situational context cues, were higher than in sets 1-2 with mere facial expressions. 
Neuropsychological examination and experimental procedures were always performed at the same hours in the morning.

\section{- Statistical Analyses}

Analyses were performed using IBM SPSS v. 23 (IBM Corp., New York, USA). The alpha level was set at $\mathrm{P}<0.05$. Kruskall-Wallis test was used to determine whether groups differed significantly in subject's age and years of education. Groups were compared in female and male proportions by Pearson's chi squared test. The Jonckheere-Terpstra tests for ordered alternatives were used to examine the overall trends among bvFTD patients, ALS patients and controls in particular sets of emotional decoding task and neuropsychological variables. Directions of tested trends (ascending or descending) for analyzed dependent variables are presented in Table $\mathbf{1}$. Statistically significant ordered overall trends were followed by one-sided Mann-Whitney $\mathrm{U}$ tests for pairwise comparisons with $\mathrm{P}-$ values adjusted with Holm's correction. This approach allowed determining whether groups differ in a specific (ascending or descending) direction, defined according to our hypotheses. Spearman's rank-order correlation coefficients were computed to assess the associations between emotional decoding and neuropsychological variables. One-tailed $\mathrm{P}$-values for correlation coefficients were calculated because specified directions of examined relationship between variables were defined. For each group the correction of $\mathrm{P}$-values for false discovery rate using the Benjamini-Hochberg method was applied.

\section{Results}

Patients from the three groups did not differ significantly regarding age, gender, years of education and disease severity. Mean ALSFRS-R score was $33.63( \pm 4.69)$ and FRS score was -.20 ( \pm 1.25$)$. Mean MOCA score for controls, ALS patients and bvFTD patients was 27.26, 26.60, and 22.85 respectively; Kruskal-Wallis $\chi^{2}(2)=16.156, p<.001$.

Comparisons of emotional decoding and executive variables are shown in Table $\mathbf{1}$. The Jonckheere-Terpstra test results revealed significant ascending differences in all emotional decoding variables among bvFTD patients, ALS patients and control subjects. Pairwise comparisons indicated that control subjects recognized emotional stimuli more correct than bvFTD patients in sets 2, 3, 4, 5 and 6, and more correct than ALS patients in sets 4 and 6, whereas ALS patients recognized emotional stimuli more correctly than bvFTD patients in set 2, 3, 4 and 5. Significant ascending trends among bvFTD patients, ALS patients and control subjects was also confirmed by the Jonckheere-Terpstra test results in regard to scores obtained in MoCA, digits forward and backwards, proverb interpretation, similarities, fonological verbal fluency, and Tower of London-number of correct task. Control subjects and ALS patients scored significantly better than bvFTD patients on all these neuropsychological tests, and control subjects scored significantly better than ALS patients on MoCA, proverb interpretation, similarities, and counting backwards by 7 tests. The Jonckheere-Terpstra test results confirmed significant descending trends among bvFTD patients, ALS patients and control subjects in Tower of London-number of movements, time needed to finish, rules violation, Trial Making Test part B/A and Stroop Test (interference task). In all these variables significantly lower values were found in control subjects and ALS patients compared to bvFTD patients. In Trial Making Test part $\mathrm{B} / \mathrm{A}$ values were significantly lower in controls than in ALS patients.

Spearman's rank-order correlation coefficients between emotional decoding variables and neuropsychological test scores are presented in Table 2. Total numbers of significant correlations between correctness of emotional decoding and all neuropsychological variables were calculated separately for bvFTD patients, ALS patients and control subjects to summarize results presented in Table 2. In bvFTD patients no significant correlations was found for set 1,2 and 3 , whereas 7,5 and 1 correlations reached the level of significance respectively for set 4 , 5 and 6. In ALS patients 1, 8, 7, 6, 5, and 2 significant correlations was found for set 1 to 6 , respectively. In control subjects the only significant correlation was found between set 6 of emotional decoding task and Tower of London - number of movements. The percentages of all significant correlations in bvFTD patients, ALS patients and control subjects were 16.7, 37.2, and 1.3 respectively.

\section{Discussion}

This study aimed to compare ability of bvFTD, 
Emotions processing in amyotrophic lateral sclerosis and behavioral variant of frontotemporal dementia- Research correlation with executive functions

\begin{tabular}{|c|c|c|c|c|c|c|c|}
\hline \multirow{2}{*}{ Variable } & \multicolumn{3}{|c|}{$\begin{array}{c}\text { Medians } \\
\left(25^{\text {th }}: 75^{\text {th }} \text { percentiles }\right)\end{array}$} & \multicolumn{2}{|c|}{ Jonckheere-Terpstra test } & \multirow{2}{*}{$P$} & \multirow{2}{*}{$\begin{array}{l}\text { Pairwise } \\
\text { comparisons }\end{array}$} \\
\hline & bvFTD & ALS & Controls & $\begin{array}{l}\text { ordering } \\
\text { direction }\end{array}$ & $\begin{array}{l}\text { observed } \\
\text { value }\end{array}$ & & \\
\hline Set 1 & $\begin{array}{c}3.5 \\
(2.0: 5.0)\end{array}$ & $\begin{array}{c}4.0 \\
(2.75: 5.0)\end{array}$ & $\begin{array}{c}4.0 \\
(3.0: 6.0)\end{array}$ & ascending & 1909.5 & 0.0225 & - \\
\hline Set 2 & $\begin{array}{c}3.5 \\
(2.0: 4.0)\end{array}$ & $\begin{array}{c}4.0 \\
(3.0: 6.0)\end{array}$ & $\begin{array}{c}5.0 \\
(3.0: 6.0)\end{array}$ & ascending & 2040.0 & 0.0022 & $a, b$ \\
\hline Set 3 & $\begin{array}{c}3.0 \\
(2.0: 4.25)\end{array}$ & $\begin{array}{c}5.0 \\
(4.0: 6.0)\end{array}$ & $\begin{array}{c}5.0 \\
(4.0: 5.0)\end{array}$ & ascending & 2104.5 & 0.0005 & $a, b$ \\
\hline Set 4 & $\begin{array}{c}3.0 \\
(2.0: 5.0)\end{array}$ & $\begin{array}{c}5.0 \\
(3.0: 6.0)\end{array}$ & $\begin{array}{c}6.0 \\
(5.0: 7.0)\end{array}$ & ascending & 2312.0 & 0.0002 & $a, b, c$ \\
\hline Set 5 & $\begin{array}{c}3.0 \\
(2.0: 5.0)\end{array}$ & $\begin{array}{c}5.0 \\
(4.0: 6.0)\end{array}$ & $\begin{array}{c}5.0 \\
(5.0: 6.0)\end{array}$ & ascending & 2241.5 & $<0.001$ & $a, b$ \\
\hline Set 6 & $\begin{array}{c}3.0 \\
(2.0: 4.0)\end{array}$ & $\begin{array}{c}3.5 \\
(2.0: 4.0)\end{array}$ & $\begin{array}{c}4.0 \\
(3.0: 5.0)\end{array}$ & ascending & 2138.5 & 0.0002 & $b, c$ \\
\hline MoCA & $\begin{array}{c}23.0 \\
(19.0: 25.25)\end{array}$ & $\begin{array}{c}28.0 \\
(26.75: 29.0)\end{array}$ & $\begin{array}{c}29.0 \\
(28.0: 30.0)\end{array}$ & ascending & 2558.0 & 0.000 & $a, b, c$ \\
\hline Digits forward & $\begin{array}{c}5.0 \\
(4.0: 5.0)\end{array}$ & $\begin{array}{c}5.0 \\
(5.0: 6.0)\end{array}$ & $\begin{array}{c}5.0 \\
(5.0: 6.0)\end{array}$ & ascending & 1850.5 & 0.007 & $a, b$ \\
\hline Digits backward & $\begin{array}{c}3.0 \\
(3.0: 4.0)\end{array}$ & $\begin{array}{c}4.0 \\
(3.0: 4.0)\end{array}$ & $\begin{array}{c}4.0 \\
(3.0: 4.0)\end{array}$ & ascending & 1956.0 & 0.001 & $a, b$ \\
\hline Proverb interpretation & $\begin{array}{c}4.0 \\
(2.0: 6.0)\end{array}$ & $\begin{array}{c}8.0 \\
(6.0: 8.0)\end{array}$ & $\begin{array}{c}8.0 \\
(8.0: 8.0)\end{array}$ & ascending & 2142.0 & $<0.001$ & $a, b, c$ \\
\hline Similarities & $\begin{array}{c}7.0 \\
(4.0: 9.0)\end{array}$ & $\begin{array}{c}11.0 \\
(8.0: 12.0)\end{array}$ & $\begin{array}{c}11.0 \\
(10.0: 12.0)\end{array}$ & ascending & 2076.5 & $<0.001$ & $a, b, c$ \\
\hline Verbal fluency (K) & $\begin{array}{c}6.0 \\
(3.5: 9.5)\end{array}$ & $\begin{array}{c}12.0 \\
(9.0: 17.5)\end{array}$ & $\begin{array}{c}13.0 \\
(11.0: 16.0)\end{array}$ & ascending & 2092.5 & $<0.001$ & $a, b$ \\
\hline $\begin{array}{l}\text { Tower of London number of } \\
\text { correct task }\end{array}$ & $\begin{array}{c}2.0 \\
(1.0: 4.0)\end{array}$ & $\begin{array}{c}3.0 \\
(2.0: 5.0)\end{array}$ & $\begin{array}{c}4.0 \\
(2.0: 5.0)\end{array}$ & ascending & 1791.5 & 0.002 & $b$ \\
\hline $\begin{array}{l}\text { Tower of London -number of } \\
\text { movements }\end{array}$ & $\begin{array}{c}47.0 \\
(27.0: 49.0)\end{array}$ & $\begin{array}{c}35.0 \\
(24.75: 44.75)\end{array}$ & $\begin{array}{c}27.0 \\
(18.0: 44.0)\end{array}$ & descending & 1142.5 & 0.042 & d, e \\
\hline $\begin{array}{l}\text { Tower of London time needed } \\
\text { to finish }\end{array}$ & $\begin{array}{c}565.0 \\
(365.0: 922.0)\end{array}$ & $\begin{array}{c}311.0 \\
(220.0: 390.5)\end{array}$ & $\begin{array}{c}253.0 \\
(182.0: 357.0)\end{array}$ & descending & 769.0 & $<0.001$ & $d, e$ \\
\hline $\begin{array}{c}\text { Tower of London - rules } \\
\text { violation }\end{array}$ & $\begin{array}{c}5.0 \\
(1.0: 8.0)\end{array}$ & $\begin{array}{c}0.5 \\
(0.0: 1.3)\end{array}$ & $\begin{array}{c}0.0 \\
(0.0: 1.0)\end{array}$ & descending & 899.0 & $<0.001$ & $d, e$ \\
\hline Trial Making Test part B/A & $\begin{array}{c}1.82 \\
(1.39: 3.29)\end{array}$ & $\begin{array}{c}1.55 \\
(1.20: 2.01)\end{array}$ & $\begin{array}{c}1.48 \\
(1.26: 1.82)\end{array}$ & descending & 972.5 & 0.030 & $d, e, f$ \\
\hline $\begin{array}{l}\text { Stroop Test (number of errors } \\
\text { in interference task) }\end{array}$ & $\begin{array}{c}3.0 \\
(0.0: 9.75)\end{array}$ & $\begin{array}{c}0.0 \\
(0.0: 1.0)\end{array}$ & $\begin{array}{c}0.0 \\
(0.0: 1.0)\end{array}$ & descending & 711.0 & 0.013 & $d, e$ \\
\hline Counting backwards by 7 & $\begin{array}{c}3.0 \\
(1.0: 5.0)\end{array}$ & $\begin{array}{c}5.0 \\
(3.0: 5.0)\end{array}$ & $\begin{array}{c}5.0 \\
(5.0: 5.0)\end{array}$ & ascending & 2347.5 & $<0.001$ & $a, b, c$ \\
\hline
\end{tabular}

ALS patients and controls to discriminate emotions. Control subjects decoded emotions with the highest accuracy, while bvFTD patients decoded emotions with the lowest accuracy. The ability to decode emotions differed in particular task.

Patients with bvFTD decoded emotions with the lowest accuracy, significantly worse than patients with ALS (4/6 sets) and control subject (5/6 sets). Patients with ALS decoded emotions worse than controls (significantly worse on $2 / 6$ sets). The accuracy of decoding in patients with ALS was very similar to controls but different to the group of patients with bvFTD (with medians in the following sets: $4,5,5,6,5,4$ in controls; 4, 4, 5, 5, 5, 3.5 in ALS and 3.5, 3.5, 3, 3, 3, 3 bvFTD). Emotions were decoded on sets 3,4 , and 5 with highest accuracy in the group of patients with ALS and on sets 2,3,4 and 5 in control subjects. In bvFTD the accuracy of decoding abilities was described by almost a flat line, what's more, in set 3,4 and 5 the accuracy of decoding emotions was worse than in sets 1 and 2 , what was in contrast to other groups.

Pictures with standardized facial expressions are thought to poorly reflect the context of the natural environment. Stimuli depicting more realistic emotional situations may enrich the 
Research Aleksandra Klimkowicz-Mrowiec

\begin{tabular}{|c|c|c|c|c|c|c|c|c|c|c|c|c|c|c|c|c|c|c|}
\hline \multirow{2}{*}{ Variables } & \multicolumn{6}{|c|}{ bvFTD } & \multicolumn{6}{|l|}{ ALS } & \multicolumn{6}{|c|}{ Controls } \\
\hline & Set 1 & Set 2 & Set 3 & Set 4 & Set 5 & Set 6 & Set 1 & Set 2 & Set 3 & Set 4 & Set 5 & Set 6 & Set 1 & Set 2 & Set 3 & Set 4 & Set 5 & Set 6 \\
\hline MoCA & .138 & .224 & .399 & $.605^{*}$ & $.475^{*}$ & .344 & $.350 *$ & $.471^{*}$ & $.338^{*}$ & $.553^{* *}$ & .241 & $.347^{*}$ & .062 & .065 & .312 & .212 & .382 & .016 \\
\hline Digits forward & -.427 & -.412 & -.083 & -.037 & -.100 & -.209 & .096 & $.424^{*}$ & $.406^{*}$ & $.422^{*}$ & $.376^{*}$ & .140 & .100 & .104 & -.010 & .202 & -.022 & .095 \\
\hline Digits backward & -.040 & .127 & .250 & .310 & .261 & .487 & 292 & $.319^{*}$ & .217 & .280 & $.397^{*}$ & $.416^{*}$ & .280 & .129 & .010 & .437 & .100 & .279 \\
\hline $\begin{array}{l}\text { Proverb } \\
\text { interpretation }\end{array}$ & .291 & .105 & .402 & $.552^{*}$ & $.591^{*}$ & .372 & .281 & .100 & $.373^{*}$ & $.467^{*}$ & .290 & .187 & .170 & .253 & .134 & .172 & .010 & -.118 \\
\hline Similarities & .324 & .369 & .390 & $.597^{*}$ & $.527^{*}$ & $.623^{*}$ & .317 & $.382^{*}$ & $.498^{*}$ & .322 & .260 & .135 & -.101 & -.042 & -.083 & -.256 & -.143 & -.301 \\
\hline Verbal fluency K & .473 & .182 & .208 & .222 & .261 & .397 & $.383^{*}$ & .192 & .265 & $.513^{* *}$ & $.392^{*}$ & .242 & .108 & .040 & .142 & .134 & .147 & -.120 \\
\hline $\begin{array}{l}\text { Tower of London } \\
\text { number of correct } \\
\text { task }\end{array}$ & .010 & .076 & -.040 & .227 & .284 & .133 & .192 & .279 & .162 & .223 & -.038 & -.059 & .120 & .124 & .016 & .241 & -.021 & .205 \\
\hline $\begin{array}{l}\text { Tower of London } \\
\text {-number of } \\
\text { movements }\end{array}$ & .085 & -.100 & .021 & -.401 & -.411 & -.046 & -.072 & $-.404^{*}$ & -.184 & -.106 & .021 & .025 & -.043 & -.071 & -.224 & -.134 & .009 & -.300 \\
\hline $\begin{array}{l}\text { Tower of London } \\
\text { time needed to } \\
\text { finish }\end{array}$ & -.197 & -.114 & -.335 & -.514 & $-.570^{*}$ & $-.371^{*}$ & -.228 & $-.367^{*}$ & $-.366^{*}$ & -.324 & -.193 & -.264 & -.292 & -.375 & -.262 & -.504 & -.311 & $-.626^{* *}$ \\
\hline $\begin{array}{l}\text { Tower of London } \\
\text {-rules violation }\end{array}$ & -.116 & .377 & -.231 & -.225 & -.467 & .190 & $-.377^{*}$ & $-.480^{*}$ & -.130 & -.281 & $-.368^{*}$ & -.297 & .026 & -.200 & -.120 & -.124 & -.082 & -.353 \\
\hline $\begin{array}{l}\text { Trial Making Test } \\
\text { part B/A }\end{array}$ & .240 & .415 & -.216 & .100 & -.275 & .139 & -.166 & -.075 & $-.343^{*}$ & $-.339 *$ & $-.374^{*}$ & -.303 & -.161 & -.140 & -.115 & -.218 & -.028 & -.211 \\
\hline $\begin{array}{l}\text { Stroop Test } \\
\text { (number of errors } \\
\text { in task 3) }\end{array}$ & -.242 & -.179 & -.198 & $-.579^{*}$ & $-.703^{*}$ & -.222 & -.037 & -.286 & -.105 & -.132 & -.384 & .024 & -.453 & .052 & -.131 & -.374 & -.287 & -.148 \\
\hline $\begin{array}{l}\text { Counting } \\
\text { backwards by } 7\end{array}$ & .051 & .157 & .298 & $.495^{*}$ & .404 & .142 & $.343^{*}$ & $.428^{*}$ & $.383^{*}$ & $.555^{* *}$ & .182 & .238 & -.066 & .145 & -.180 & .039 & -.092 & -.181 \\
\hline
\end{tabular}

input properties and enhance the perception of emotion [37]. Patients with cognitive impairment may be more affected by ecological value of the stimuli than healthy subjects.

We observed that deficit in emotional decoding abilities in all groups was related to task complexity. Less complex stimuli were more difficult to decode than more complex.

As each task increased in its level of perceptual complexity, from the iconic figures (lowest degree of complexity and information), to more realistic pictures (showing situational drawings providing additional channel for emotional recognition i.e. gestures, context), control subjects and ALS patients demonstrated a trend of increasing accuracy, with the relapse in the last set where stimuli were presented by personified birds. Using birds, as a carriers of emotional information, occurred the most difficult to recognize for all participants, regardless of the group. In sets 1-5, with increase of ecological value of the stimuli, the increase of emotional decoding ability was observed. Patients with bvFTD did not benefit from additional hints.
In study by Lillo, et al. [22] controls performed better than ALS and bvFTD groups. There was also a significant difference between ALS and bvFTD groups (Controls , ALS , bvFTD). In the study performed by Savage, et al. [14] comparing ALS and FTD patients versus control subjects on emotional recognizing task, emotion processing deficit was present in a subset of patients with ALS, and the emergence of deficits was strongly related to the presence of FTD.

Patients with combined ALS and FTD showed disturbances in emotional recognition as severe as those seen in bvFTD, which were not explained by overall changes in cognitive ability. Authors concluded that emotion processing deficits are specific to certain subgroups of ALS patients, and arise most clearly in patients with an FTD diagnosis [14].

The direct comparison of our study and study by Lillo et al. [22] and Savage et al. [14] is impossible because of the different methodology used in all three studies. Our results support the data, those patients with ALS and controls decode emotions on a similar level, whereas patients with bvFTD 

correlation with executive functions

are significantly impaired. This might suggest that deficit in emotional decoding abilities in ALS patients is more due to orbitofrontal cortex disruption than prefrontal limbic pathway.

Studies analyzing emotional decoding abilities in psychiatric disorders found that facial emotion recognition impairment correlated with executive impairment in people with schizophrenia [18] and their unaffected siblings but not in healthy controls [19].

Therefore in our study we hypothesized those worse emotional decoding abilities may be related to worse executive functioning. To check this hypothesis we compared all groups on executive measures and we found that all groups differed significantly on most of the analyzed data. (Table 2 shows the results). Relative to control subjects, patients with bvFTD performed significantly worse on all executive measures, and relative to patients with ALS performed significantly worse on proverb interpretation, similarities and Stroop Test.

When we correlated executive functioning measures with decoding abilities within each group, we found that executive impairment correlated with worse emotional decoding abilities, particularly in ALS patients. In the group of patients with ALS there were twice as many significant correlations then in the group of patients with bvFTD group, and only 1 out of 78 (1.3\%) in the control group. Patients with ALS showed negative correlations between executive measures and emotional decoding ability along all 6 sets, mainly in sets 2 and 3. Patients with bvFTD showed negative correlations between executive measures and emotional decoding ability only in sets 4 and 5. In the control group only one positive correlation was detected on set 6 . The following sets, being more and more complex engage more cognitive resources and executive control to make a proper judgment. Worse executive functioning may affect ability to properly understand the situation and recognize emotions. Impairment in executive functioning according to expectations exacerbated the accuracy of emotional decoding ability in bvFTD. In ALS patients, in contrast what we thought, regardless of the stimuli complexity, impaired decoding correlated with impaired executive measures.

This result does not support the hypothesis of common continuum, but rather the hypothesis of divergent neuropathological background.

Although all patients underwent MR or CT neuroimaging, we could not compare the size of atrophy with emotional recognition ability, which presented a limitation of this study. We also did not analyze the differences in ability to recognize particular emotion among studied groups; therefore the final conclusion regarding the pattern of emotional stimuli recognition between groups is not possible. We hope to continue our study, and include patients with ALS-FTD to compare them with other groups.

\section{Conclusion}

Patients with bvFTD decode emotions significantly worse than ALS and controls.

The complexity of emotional stimuli affects the accuracy of emotional decoding ability.

Executive dysfunction correlates negatively with emotional decoding abilities in patients with ALS, less with bvFTD.

\section{Acknowledgements \\ This study was supported by Jagiellonian University KIZDS/005600.}

\section{Conflict of interest}

The authors declare no conflict of interest.

\section{References}

1. Brooks BR, Miller RG, Swash M, et al. World Federation of Neurology Research Group on Motor Neuron Diseases. El Escorial revisited: revised criteria for the diagnosis of amyotrophic lateral sclerosis. Amyotroph. Lateral Scler. Other. Motor. Neuron. Disord 1(5), 293-299 (2000).

2. Abrahams S, Goldstein LH, Al-Chalabi A, et al. Relation between cognitive dysfunction and pseudobulbar palsy in amyotrophic lateral sclerosis. J. Neurol. Neurosurg. Psychiatry 62(5), 464-472 (1997).

3. Lomen-Hoerth C, Murphy J, Langmore $\mathrm{S}$, et al. Are amyotrophic lateral sclerosis patients cognitively normal? Neurology 60(7), 1094-1097 (2003).

4. Ringholz GM, Appel SH, Bradshaw M, et al. Prevalence and patterns of cognitive impairment in sporadic ALS. Neurology 23(4), 586-590 (2005).

5. Murphy JM, Henry RG, Langmore S, et al.
Continuum of frontal lobe impairment in amyotrophic lateral sclerosis. Arch. Neurol 64(4), 530-534 (2007).

6. Harciarek M, Cosentino S. Language, executive function and social cognition in the diagnosis of frontotemporal dementia syndromes. Int. Rev. Psychiatry 25(2), 178196 (2013).

7. Yokoyama JS, Sirkis DW, Miller BL. C9ORF72 hexanucleotide repeats in behavioral and motor neuron disease: clinical 
heterogeneity and pathological diversity. Am. J. Neurodegener. Dis 3(1), 1-18 (2014).

8. Neumann M, Kwong LK, Sampathu DM, et al. TDP-43 proteinopathy in frontotemporal lobar degeneration and amyotrophic lateral sclerosis: protein misfolding diseases without amyloidosis. Arch. Neurol 64(10), 1388-1394 (2007).

9. Giordana MT, Ferrero P, Grifoni S, et al. Dementia and cognitie impairment in amyotrophic lateral sclerosis: a review. Neurol. Sci 32(1), 9-16 (2011).

10. Phukan J, Pender NP, Hardiman O. Dementia and cognitive impairment in amyotrophic lateral sclerosis: a review. Lancet. Neurol 6(11), 994-1003 (2011).

11. Machts J, Loewe K, Kaufmann J, et al. Basal ganglia pathology in ALS is associated with neuropsychological deficits. Neurology 13(85), 1301-1309 (2015).

12. Fusar-Poli $P$, Placentino $A$, Carletti $F$, et al. Functional atlas of emotional faces processing: a voxel-based meta-analysis of 105 functional magnetic resonance imaging studies. J. Psychiatry Neurosci 34(6), 418-432 (2009).

13. Hsieh S, Hornberger M, Piguet O, et al. Brain correlates of musical and facial emotion recognition: evidence from the dementias. Neuropsychologia 50(8), 1814-1822 (2012).

14. Savage SA, Lillo P, Kumfor F, et al. Emotion processing deficits distinguish pure amyotrophic lateral sclerosis from frontotemporal dementia. Amyotroph. Lateral Scler. Frontotemporal Degener 15(12), 39-46 (2014).

15. Zimmerman EK, Eslinger PJ, Simmons $Z$, et al. Emotional perception deficits in amyotrophic lateral sclerosis. Cogn. Behav. Neurol 20(2), 79-82 (2007).

16. Girardi A, Macpherson SE, Abrahams S. Deficits in emotional and social cognition in amyotrophic lateral sclerosis. Neuropsychology 25(1), 53-65 (2011).

17. App B, Mclntosh DN, Reed CL, et al. Nonverbal channel use in communication of emotion: how may depend on why. Emotion 11(3), 603-617 (2011).
18. Yang C, Zhang T, Li Z, et al. The relationship between facial emotion recognition and executive functions in first-episode patients with schizophrenia and their siblings. BMC. Psychiatry 8(15), 241-249 (2015).

19. Kohler CG, Bilker W, Hagendoorn $M$, et al. Emotion recognition deficit in schizophrenia: association with symptomatology and cognition. Biol. Psychiatry 48(2), 127-36 (2000).

20. Hoaken PN, Allaby DB, Earle J. Executive cognitive functioning and the recognition of facial expressions of emotion in incarcerated violent offenders, non-violent offenders, and controls. Aggress. Behav 33(5), 412-421 (2007)

21. Phillips ML, Drevets WC, Rauch SL, et al. Neurobiology of emotion perception I: The neural basis of normal emotion perception. Biol. Psychiatry 54(5), 504-514 (2003).

22. Lillo P, Savage S, Mioshi E, et al. Amyotrophic lateral sclerosis and frontotemporal dementia: A behavioural and cognitive continuum. Amyotroph. Lateral. Scler 13(1), 102-109 (2012).

23. Mattis S. Mental status examination for organic mental syndrome in the elderly patients. In: Geriatric Psychiatry: A Handbook for Psychiatrists and Primary Care Physicians. (ed. L. Bellak and T.B. Karasu), Grune \& Stratton: New York 77-12 (1976).

24. Lezak MD. Neuropsychological assessment. 2nd ed. Oxford University Press: New York (1982).

25. Reitan RM. Validity of the trail making test as on indicator of organic brain damage. Perception. Motor Skills 8(1), 271-276 (1958).

26. Shallice T. Specific impairments of planning. Philosophical Transactions of the Royal Society of London. Biol. Sci 298(1), 199-209 (1982).

27. Stroop JR. Studies of interference in serial verbal reactions. J. Experiment. Psychol 18(5), 643-662 (1935).

28. Nasreddine ZS, Phillips NA, Bédirian V, et al. The Montreal Cognitive Assessment, MoCA: a brief screening tool for mild cognitive impairment. J. Am. Geriatr. Soc 53(4), 695699 (2005).

29. Delis DC, Kramer JH, Kaplan E, et al. California Verbal Learning Test. Second Edition. Psychological Corporation: San Antonio, TX (1987). Polish adaptation by Lojek E, Stanczak J. Warsaw, Poland; Psychological Test Laboratory of the Polish Psychological Association (2011).

30. Rascovsky K, Hodges JR, Knopman DS, et al. Sensitivity of revised diagnostic criteria for the behavioral variant of frontotemporal dementia. Brain 134(11), 2456-2477 (2011).

31. Mioshi E, Hsieh S, Savage S, et al. Clinical staging and disease progression in frontotemporal dementia. Neurology 18(20), 1591-1597 (2010).

32. Steketee RM, Meijboom R, Bron EE, et al. Structural and functional brain abnormalities place phenocopy frontotemporal dementia (FTD) in the FTD spectrum. Neuroimage. Clin 10(4), 595-605 (2016).

33. Cedarbaum JM, Stambler N, Malta E, et al. The ALSFRS-R: a revised ALS functional rating scale that incorporates assessments of respiratory function. BDNF ALS Study Group (Phase III). J. Neurol. Sci 31(1-2), 13-21 (1999).

34. Yesavage JA. Geriatric Depression Scale. Psychopharmacol. Bull 24(4), 709-711 (1988)

35. Klimkowicz-Mrowiec A, Krzywoszanski L Spisak K, et al. Emotional decoding abilities do not influence behavioral disturbances in Alzheimer's disease. J. Am. Geriatr. Soc 61(3), 454-456 (2013).

36. Klimkowicz-Mrowiec A., Krzywoszanski L, Spisak K, et al. A Comparison of Emotional Decoding Abilities in Patients with Amnestic Mild Cognitive Impairment, Very Mild and Mild Alzheimer's Disease. Current. Alzheimer. Res 11(2), 200-205 (2014).

37. Ambadar Z, Schooler JW, Cohn JF. Deciphering the enigmatic face: the importance of facial dynamics in interpreting subtle facial expressions. Psychol. Sci 16(5), 403-410 (2005). 